\title{
Sum rules for tetraquark decay coupling constants with broken SU(3) symmetry
}

\author{
V. Guptat and G. Sánchez-Colónđ \\ Departamento de Física Aplicada. \\ Centro de Investigación y de Estudios Avanzados del IPN. \\ Unidad Mérida. \\ A.P. 73, Cordemex. \\ Mérida, Yucatán, 97310. MEXICO. \\ S. Rajpoot \\ Department of Physics \& Astronomy. \\ California State University, Long Beach. \\ Long Beach, CA 90840. USA.
}

(Dated: August 11, 2018)

\begin{abstract}
We give sum rules for tetraquark decay coupling constants, taking into account the SU(3) symmetry breaking interactions to first order.
\end{abstract}

PACS numbers: 14.40.Rt, 13.25.Jx

Keywords: tetraquarks, sum rule

*virendra@mda.cinvestav.mx

gsanchez@mda.cinvestav.mx

rajpoot@csulb.edu 


\section{INTRODUCTION}

Recently, two hidden-bottom charged meson resonances, referred to as $Z_{b}(10610)$ and $Z_{b}(10650)$, were observed by the Belle Collaboration [1] as two narrow resonance structures in the mass spectra of $\pi^{ \pm} \Upsilon(n S)(n=1,2,3)$ and $\pi^{ \pm} h_{b}(m P)(m=1,2)$. They are produced in association with a single charged pion in $\Upsilon(5 S)$ decays with the following values of mass and width, averaged over the five final states:

$$
\begin{aligned}
& M\left[Z_{b}(10610)\right]=(10607.2 \pm 2.0) \mathrm{MeV} / c^{2}, \quad \Gamma\left[Z_{b}(10610)\right]=(18.4 \pm 2.4) \mathrm{MeV}, \\
& M\left[Z_{b}(10650)\right]=(10652.2 \pm 1.5) \mathrm{MeV} / c^{2}, \quad \Gamma\left[Z_{b}(10650)\right]=(11.5 \pm 2.2) \mathrm{MeV}
\end{aligned}
$$

Two main theoretical structure assignments are proposed for these hidden-bottom meson resonances. One is based on molecular excitations [2, 3], the molecules being two meson bound state with a very small binding energy, and the other one is the tetra-quark interpretation [4, 5] using the analogy similar to the charm sector.

The $J^{P}$ of both, $Z_{b}(10610)$ and $Z_{b}(10650)$, is determined to be $1^{+}$. This is consistent with their decay into a $1^{-}$-state $(\Upsilon(n S))$ and a $0^{-}$-meson in a relative $s$-state. In this note we suggest that these new states are tetraquarks made of $(b \bar{b} q \bar{q})$ with $q=u, d, s$. We work out the consequences of broken SU(3) symmetry to derive sum rules for tetraquark decay coupling constants.

\section{NOTATION}

Let us consider a general tetraquark $T(Q \bar{Q} q \bar{q}))$ with $Q(=b$ or $c)$ quark a $\mathrm{SU}(3)$ flavour singlet and $q \bar{q}$ made of the light quarks $q(=u, d$, and $s)$. One natural decay mode of the tetraquark $T(Q \bar{Q} q \bar{q})$ would be

$$
T(Q \bar{Q} q \bar{q}) \rightarrow H(Q \bar{Q})+M(q \bar{q})
$$

where $H(Q \bar{Q})$ is a heavy meson since $Q=b$ or $c$ and $M(q \bar{q})$ could be the $\mathrm{SU}(3)$ flavour $0^{-}$ pseudoscalar octet $\mathbf{P}$,

$$
\mathbf{P}=\left(\begin{array}{ccc}
\frac{1}{\sqrt{2}} \pi^{0}+\frac{1}{\sqrt{6}} \eta & \pi^{+} & K^{+} \\
\pi^{-} & -\frac{1}{\sqrt{2}} \pi^{0}+\frac{1}{\sqrt{6}} \eta & K^{0} \\
K^{-} & \bar{K}^{0} & -\frac{2}{\sqrt{6}} \eta
\end{array}\right)
$$

or the $1^{-}$vector octet $\mathbf{V}$,

$$
\mathbf{V}=\left(\begin{array}{ccc}
\frac{1}{\sqrt{2}} \rho^{0}+\frac{1}{\sqrt{2}} \omega & \rho^{+} & K^{*+} \\
\rho^{-} & -\frac{1}{\sqrt{2}} \rho^{0}+\frac{1}{\sqrt{2}} \omega & K^{* 0} \\
K^{*-} & \bar{K}^{* 0} & \phi
\end{array}\right)
$$

Furthermore, one expects that $H(Q \bar{Q})$ and $M(q \bar{q})$ in the final state have the same $J^{P}$ they have in the tetraquark. This is the simplest possibility. 


\section{SUM RULES}

If one assumes that the decay interaction is flavour $\mathrm{SU}(3)$ singlet, then all the eight decays will have the same coupling constant $G_{0}$. However if one considers a first order breaking interaction transforming like $\lambda_{8}$ (or $T_{3}^{3}$ ) which conserves isospin invariance then this will give rise to two $\mathrm{SU}(3)$ breaking coupling constants, $G_{F}$ and $G_{D}$, which arise from the antisymmetric and symmetric octets resulting from the breaking interaction and the $M(q \bar{q})$ octet in the final state. Thus, the eight decays will be given in terms of three coupling constants. However, since isospin invariance is present, there will be four independent coupling constants namely, when the $M(q \bar{q})$ meson is $\left(K^{+}, K^{0}\right),\left(\bar{K}^{0}, K^{-}\right),\left(\pi^{+}, \pi^{0}, \pi^{-}\right)$, and $s \bar{s}$. These four coupling constants will be linear combinations of $G_{0}, G_{F}$, and $G_{D}$, and hence, we expect a sum rule among the four coupling constants of the observable decays [6 8].

Since the SU(3) calculation is analogous to the calculation of the meson masses with $\lambda_{8}$ breaking, one obtains a coupling constants sum rule analogous to the Gell-Mann-Okubo mass formula for mesons [9, 10]. Thus, if our interpretation of the observed particles as tetraquarks is right we expect,

$$
\begin{aligned}
2 G[T(Q \bar{Q} K) & \rightarrow H(Q \bar{Q})+K]+2 G[T(Q \bar{Q} K) \rightarrow H(Q \bar{Q})+\bar{K}]= \\
G[T(Q \bar{Q} \pi) & \rightarrow H(Q \bar{Q})+\pi]+3 G[T(Q \bar{Q} s \bar{s}) \rightarrow H(Q \bar{Q})+M(s \bar{s})] .
\end{aligned}
$$

Here $K=K^{+}$or $K^{0}, \bar{K}=\bar{K}^{0}$ or $K^{-}, \pi=\pi^{+}, \pi^{-}$, or $\pi^{0}$. The heavy quark $Q$ is a $\mathrm{SU}(3)$ singlet and could be either $b$ or $c$ quark.

The above formula for decay coupling constants is also valid for tetraquarks $T\left(Q \bar{Q}^{\prime} M(q \bar{q})\right)$ where $Q=b$ (or $\left.c\right)$ with $Q^{\prime}=c($ or $b)$.

As noted earlier, the light quark octet $M(q \bar{q})$ could be the $1^{-}$vector octet $\left(\rho, K^{*}\right.$, etc.). In this case, the sum rules would be valid with the obvious changes $\pi \rightarrow \rho, K \rightarrow K^{*}$, etc. The sum rules contain $M(s \bar{s})$ and represents $\eta, \eta^{\prime}, \phi$ or $\omega$ as appropriate.

Applied to specific decay modes of the tetraquarks, the sum rule translates into the following sum rules,

$$
\begin{aligned}
2 G_{K^{+}}+2 G_{\bar{K}^{0}} & =G_{\pi^{+}}+3 G_{\eta}, \\
2 G_{K^{-}}+2 G_{K^{0}} & =G_{\pi^{-}}+3 G_{\eta}, \\
2 G_{K^{0}}+2 G_{\bar{K}^{0}} & =G_{\pi^{0}}+3 G_{\eta}, \\
2 G_{K^{+}}+2 G_{\bar{K}^{0}} & =G_{\pi^{+}}+3 G_{\eta^{\prime}}, \\
2 G_{K^{-}}+2 G_{K^{0}} & =G_{\pi^{-}}+3 G_{\eta^{\prime}}, \\
2 G_{K^{0}}+2 G_{\bar{K}^{0}} & =G_{\pi^{0}}+3 G_{\eta^{\prime}},
\end{aligned}
$$

where we have abbreviated the couplings as $G\left[T\left(Q \bar{Q} K^{+}\right) \rightarrow H(Q \bar{Q})+K^{+}\right] \equiv G_{K^{+}}$, and similarly for the other couplings.

As is well known, both for pseudoscalar and vector mesons, $s \bar{s}$ is a combination of the $\mathrm{SU}(3)$ singlet $\eta_{1}$ and the eighth component $\eta_{8}$. This mixing must be taken into account in testing our coupling constants sum rule. Specifically, in terms of the $\mathrm{SU}(3)$ flavor states $\eta_{1}$ and $\eta_{8}$,

$$
s \bar{s}=\frac{1}{\sqrt{3}} \eta_{1}-\frac{2}{\sqrt{6}} \eta_{8},
$$

where 


$$
\eta_{1}=\frac{1}{\sqrt{3}}(u \bar{u}+d \bar{d}+s \bar{s}) \quad \text { and } \quad \eta_{8}=\frac{1}{\sqrt{6}}(u \bar{u}+d \bar{d}-2 s \bar{s}) .
$$

For $J^{P}=0^{-}, \eta(548)=\eta_{8} \cos \theta-\eta_{1} \sin \theta$ and $\eta^{\prime}(958)=\eta_{8} \sin \theta+\eta_{1} \cos \theta$, with $\theta=-11.5^{\circ}$. For $J^{P}=1^{-}, \eta(548) \rightarrow \phi(1020)$ and $\eta^{\prime}(958) \rightarrow \omega(782)$, with $\theta=38.7^{\circ}[11]$.

The various couplings entering the sum rules can be extracted from the appropriate decay widths. For the $Z_{b}(10610)$ and $Z_{b}(10650)$ tetraquark decays into two particles, a $1^{-}$-state $(\Upsilon(n S))$ and a $0^{-}$-meson, in a relative $s$-state the width is given by

$$
\Gamma=\frac{1}{8 \pi} \frac{k}{M^{2}}|A|^{2}
$$

where $M$ is the tetraquark mass, $k$ is the momentum of a decay particle and $A$ is the transition amplitude. For $s$-wave decay we take $A=G M$, so that

$$
G^{2}=\frac{8 \pi}{k} \Gamma
$$

Numerical results for $G\left[Z_{b}(10610) \rightarrow \Upsilon(n S)+\pi^{+}\right]$and $G\left[Z_{b}(10650) \rightarrow \Upsilon(n S)+\pi^{+}\right](n=$ $1,2,3)$ are presented in Table [1] [1]. The sum rules can hopefully be verified when data for other decay modes of $Z_{b}$-tetraquarks are available.

\section{CONCLUSIONS}

In anticipation of more solid data on tetraquarks in the future [12], we have derived sum rules for tetraquark decay coupling constants assuming broken SU(3) symmetry only up to the first order. Our sum rules, Eqs. (5), rely on the assumption that the heavy quarks

are spectator singlets. It will be interesting to check these sum rules when the tetraquark spectroscopy gets better defined in the future.

\section{Acknowledgments}

V. Gupta and G. Sánchez-Colón would like to thank CONACyT (México) for partial support. The work of S. Rajpoot was supported by DOE Grant \#: DE-FG02-10ER41693.

[1] A. Bondar et al. (Belle Collaboration), Phys. Rev. Lett. 108 (2012) 122001.

[2] A. E. Bondar, A. Garmash, A. I. Milstein, R. Mizuk and M. B. Voloshin, Phys. Rev. D 84 (2011) 054010.

[3] M. B. Voloshin, Phys. Rev. D 84 (2011) 031502.

[4] T. Guo, L. Cao, M. Z. Zhou and H. Chen, "The possible candidates of tetraquark : $Z_{b}(10610)$ and $Z_{b}(10650) "$, arXiv:1106.2284 [hep-ph]].

[5] F. S. Navarra, M. Nielsen, J. -M. Richard, "Exotic Charmonium and Bottomonium-like Resonances", arXiv:1108.1230 [hep-ph]].

[6] V. Gupta and V. Singh, Phys. Rev. 135 (1964) 1442.

[7] V. Gupta and V. Singh, Phys. Rev. 136 (1964) 782. 
[8] V. Gupta, Il Nvo. Cim. 42 (1966) 737.

[9] M. Gell-Mann, "The Eightfold Way: A Theory of Strong Interaction Symmetry", California Institute of Technology Synchrotron Laboratory Report CTSL-20 (1961), unpublished.

[10] S. Okubo, Prog. Theor. Phys. 27 (1962) 949.

[11] K. Nakamura et al. (Particle Data Group), J. Phys. G. 37 (2010) 075021.

[12] S. Eidelman et al., "Developments in heavy Quark Spectroscopy", [arXiv:1205:4189 [hep-ex]]. 
TABLE I: Numerical results for $Z_{b}(10610)$ and $Z_{b}(10650)$ parameters obtained from $Z_{b}(10610) \rightarrow$ $\Upsilon(n S)+\pi^{+}$and $Z_{b}(10650) \rightarrow \Upsilon(n S)+\pi^{+}(n=1,2,3)$ analysis.

\begin{tabular}{lccc}
\hline \hline & $\Upsilon(1 S) \pi^{+}$ & $\Upsilon(2 S) \pi^{+}$ & $\Upsilon(3 S) \pi^{+}$ \\
\hline$M\left[Z_{b}(10610)\right](\mathrm{MeV})$ & $10611 \pm 4$ & $10609 \pm 2$ & $10608 \pm 2$ \\
$\Gamma\left[Z_{b}(10610)\right](\mathrm{MeV})$ & $22.3 \pm 7.7$ & $24.2 \pm 3.1$ & $17.6 \pm 3.0$ \\
$k(\mathrm{MeV})$ & $1080.3 \pm 3.6$ & $553.2 \pm 2.0$ & $208.3 \pm 2.4$ \\
$G^{2}$ & $0.52 \pm 0.18$ & $1.10 \pm 0.14$ & $2.12 \pm 0.36$ \\
\hline$M\left[Z_{b}(10650)\right](\mathrm{MeV})$ & $10657 \pm 6$ & $10651 \pm 2$ & $10652 \pm 1$ \\
$\Gamma\left[Z_{b}(10650)\right](\mathrm{MeV})$ & $16.3 \pm 9.8$ & $13.3 \pm 3.3$ & $8.4 \pm 2.0$ \\
$k(\mathrm{MeV})$ & $1121.8 \pm 5.4$ & $594.0 \pm 2.0$ & $258.3 \pm 1.2$ \\
$G^{2}$ & $0.37 \pm 0.22$ & $0.56 \pm 0.14$ & $0.82 \pm 0.19$ \\
\hline \hline
\end{tabular}

